\title{
BIENESTAR EN SISTEMAS DE ENGORDE DE CORDEROS: INDICADORES DE FISIOLÓGICOS Y DE COMPORTAMIENTO
}

\author{
LeVa, P.e. ${ }^{1} ;$ GarcíA, M.S. ${ }^{1} ;$ Fernández, G. ${ }^{2}$; Toffoli, G. ${ }^{1}$; \\ Cernotto, C. ${ }^{2} ;$ Sosa, J. ${ }^{3}$ \& Boggero, C. ${ }^{3}$
}

\begin{abstract}
RESUMEN
Durante el verano 2013 se llevó a cabo un ensayo de engorde de corderos con el objetivo de evaluar el bienestar en dos sistemas de manejo:1.- A corral; 2.- en box. Se seleccionaron aleatoriamente 8 corderos a cada sistema, manteniéndose similares características de peso y edad en cada uno. Las mediciones realizadas semanalmente y cada 4 horas fueron: Temperatura rectal y Frecuencia respiratorias, utilizándose un termómetro y un estetoscopio. Paralelamente se evaluó la ganancia de peso. Además se realizaron observaciones comportamentales: Parado y en decúbito: jadeando, rumiando en la sombra y el sol, además de conductas alimenticias El análisis de varianza fue utilizado para determinar diferencias significativas. La frecuencia respiratoria y la temperatura rectal no presentaron diferencias entre los tratamientos. Al detectarse variación horarias, se aplicó la metodología del cosinor para determinar ritmo circadiano. Para evaluar las diferencias en la ganancia de peso se utilizó la t de Student. No se detecto efecto del tratamiento sobre la ganancia de peso. Durante 3 días consecutivos y todas las semanas se realizaron observaciones de conducta con la metodología de Martín y Batenson. Se confeccionaron tablas de contingencias y se analizó con un chi cuadrado. El ajuste a ritmo circadiano, solo se observó un día en el tratamiento en box. Se detectó un efecto significativo del tratamiento y la conducta $(\mathrm{p}<0,001)$. Con los resultados alcanzados en este ensayo no nos permite inferir una diferencia entre los sistemas propuestos.

Palabras claves: Ritmos circadianos; sistema de manejo; comportamiento.
\end{abstract}

1.- Cátedra de Agrometeorología. Facultad de Ciencias Agrarias, UNL. Kreder 2805. (3080) Esperanza, provincia de Santa Fe. Email: pleva@fca.unl.edu.ar

2.- Cátedra de Anatomía y Fisiología Animal. Facultad de Ciencias Veterinarias (UNL). Kreder 2805. (3080) Esperanza, provincia de Santa Fe.

3 Cátedra de Producción Ovina. FCV (UNL).

Manuscrito recibido el 2 de julio de 2014 y aceptado para su publicación el 2 de febrero de 2015. 


\section{SUMMARY}

\section{Welfare system fattening lambs: physiological indicators and behavior} During summer 2013 conducted a test fattening lambs in order to assess welfare in both management systems: 1 . A poultry; 2 . in box. Were randomly selected eight lambs to each system, maintaining similar weight and age in each. Weekly and measurements taken every 4 hours were: rectal temperature, respiratory frequency, using a thermometer and a stethoscope. Same weight gain was evaluated. Standing and lying: In addition behavioral observations were performed panting, ruminating in the shade and sun, besides eating behaviors Analysis of variance was used to determine significant differences. The respiratory rate and rectal temperature did not differ between treatments. At the time variation detected, cosinor methodology was used to determine circadian rhythm. Student $t$ test was used to assess differences in weight gain. No treatment effect on weight gain was detected during 3 consecutive days every week behavioral observations were performed using the methodology of Martin and Bateson. They contingency tables were prepared and analyzed with chi square. Adjustment to circadian rhythm, only seen one day treatment box. A significant effect of treatment and behavior $(p<0.001)$ was detected. With the results obtained in this study does not allow us to infer a difference between the proposed systems.

Key words: Rhythm circadian; management systems; behavior.

\section{INTRODUCCION}

La tendencia mundial del mercado de carnes está orientada a poner mayor énfasis en satisfacer los requerimientos de los consumidores en términos de la calidad del producto. Por ello, hay que revalorizar los productos a través de procesos que aseguren calidad, seguridad y trazabilidad certificada, sustentabilidad de los sistemas y bienestar animal (BA), sin afectación del medio ambiente, basado en la utilización de Buenas Prácticas en los sistemas productivos, y obteniéndose una valor agregado en los productos provenientes de tales prácticas. La producción ovina no escapa a esta nueva situación.

El BA no es un concepto fácil de definir. El BA es claramente la característica de un animal individual y trata con los efectos de todos los aspectos del ambiente sobre el individuo. El BA es su estado en relación a sus intentos para afrontar su ambiente (6). Este estado incluye varias respuestas fisiológicas, de salud y conductuales. En relación a la conducta, los cambios observados en los patrones de comportamiento de los animales son los primeros indicadores de estrés. $(12,40,45)$. Por lo tanto, la utilización de etogramas ayuda a analizar el comportamiento y así crear ambientes mejores para los animales en producción $(4,21)$. Por otro lado la Farm Animal Welfare Council (14), un órgano asesor del gobierno británico en asuntos relacionados con el bienestar de los animales domésticos, propuso que el BA queda garantizado cuando se cumplen cinco requisitos (14): a) nutrición adecuada, b) sanidad adecuada, c) ausencia de incomodidad física y térmica, d) ausencia de miedo, dolor y estrés, y e) capacidad para mostrar la mayoría de conductas propias de la especie. La propuesta del FAWC se conoce habitualmente como las "cinco libertades". 